\title{
How to report multiple outcome metrics in virtual reality simulation
}

\author{
R. Rosenthal · M.W. von Websky · H. Hoffmann · M. Vitz · D. Hahnloser · H.C. Bucher · J. Schäfer
}

Received: 30 April 2015 / Accepted: 14 May 2015 / Published online: 16 June 2015

(C) Springer-Verlag Wien 2015

\begin{abstract}
Summary
Background Virtual reality (VR) simulation is increasingly used in surgical disciplines. Since VR simulators measure multiple outcomes, standardized reporting is needed.

Methods We present an algorithm for combining multiple VR outcomes into dimension summary mea-
\end{abstract}

R. Rosenthal, MD, MSc $(\bowtie) \cdot$ H. Hoffmann, MD · J. Schäfer, PhD Department of Surgery, University Hospital Basel,

Spitalstrasse 21,

4031 Basel, Switzerland

e-mail: rachel.rosenthal@unibas.ch

H. Hoffmann, MD

e-mail: henry.hoffmann@usb.ch

J. Schäfer, PhD

e-mail: juliane.schaefer@usb.ch

M. von Websky, MD

Department of Surgery, University Hospital Bonn,

Sigmund-Freud-Str. 25,

53127 Bonn, Germany

e-mail: martin@websky.de

M. Vitz, PhD

Laparoscopic Training Center,

Seestrasse 323,

8038 Zuerich-Wollishofen, Switzerland

e-mail: vitz@lapcenter.ch

D. Hahnloser, MD

Department of Visceral Surgery, University Hospital Lausanne,

Rue du Bugnon 46,

1011 Lausanne, Switzerland

e-mail: dieter.hahnloser@chuv.ch

H. Bucher, MD, MPH · J. Schäfer, PhD

Basel Institute for Clinical Epidemiology and Biostatistics,

University Hospital Basel,

Hebelstrasse 10,

4031 Basel, Switzerland

e-mail: heiner.bucher@usb.ch sures, which are then integrated into a meaningful total score. We reanalyzed the data of two VR studies applying the algorithm.

Results The proposed algorithm was successfully applied to both VR studies.

Conclusions The algorithm contributes to standardized and transparent reporting in VR-related research.

Keywords Surgical education · Laparoscopy • Virtual reality $\cdot$ Performance $\cdot$ Outcome

\section{Introduction}

Virtual reality (VR) simulation is increasingly used for surgical training and has been found to enhance operative performance [1]. VR simulators objectively measure multiple outcomes. When analyzing and reporting these data, questions to ask include: Which outcomes should be reported? How can outcomes be summarized and weighed? How can outcomes across different simulators and different studies be compared?

The aim of this article is to present and illustrate a methodological framework for reporting VR outcomes that summarizes the results from multiple outcomes into a total score. Given the increasing number of articles in the field, a standardized and transparent approach, minimizing the risk of selective outcome reporting and multiplicity issues, is of utmost importance.

\section{Material and methods}

Framework for analyzing and reporting multiple VR outcomes

VR simulators record multiple outcomes which may be grouped into different areas of interest or dimensions: 
1. "Accuracy" referring to "Efficacy", that is carrying out the task as foreseen (e.g., clipping and cutting the cystic duct), and "Safety", that is safely carrying out the task (e.g., without any electrocautery injuries),

2. "Time to complete a task", and

3. "Economy of movement", for example the instrument tip travel distance (path length). Time and economy of movement may be taken together as "efficiency" parameters.

Based on a prior expert judgment, all outcomes recorded are first grouped into these areas of interest. If several recorded outcomes represent the same skill (e.g., economy of movement [Percentage of target value] and instrument tip travel distance [cm], which is used to calculate the economy of movement), one representative outcome per skill should be chosen.

To analyze the simulator data, a four-step procedure is carried out:

1. Standardization, because outcomes may have been measured on different scales (e.g., seconds, percent),

2. Calculation of mean summary measures per dimension, that is for accuracy, time and economy of movement, summarizing all outcomes that have previously been allocated to the three dimensions,

3. Re-Standardization and unification of directionalities, so that higher values correspond to better performance throughout (e.g., instrument tip travel distance needs to be reversed (i.e., the values multiplied by minus one), because as measured by the simulator, lower distances correspond to better performance), and

4. Calculation of a weighted average as total performance score.

Standardization is a simple calculation carried out as follows: the overall mean is subtracted from each outcome and then the result is divided by the outcome's overall standard deviation [SD]. To give an example, if a group of trainees are found to have a mean time to task completion of $120 \mathrm{~s}$ with a SD of 20 and individual A of this group takes $160 \mathrm{~s}$, whereas individual $\mathrm{B}$ of this group takes $100 \mathrm{~s}$, then this results in $(160-120) / 20=2$ in individual $A$, that is he is 2 SDs slower than the mean and in $(100-120) / 20=-1$ in individual $B$, that is he is one SD faster than the mean.

To end up with positive and larger numbers to which most people are more accustomed, we suggest standardizing to mean 100 and SD 15 (rather than mean 0 and SD 1 , resulting in scores such as in the examples above of for instance minus 1 or 2). This procedure is supported by the norming procedure of many intelligence tests to mean 100 and SD 15 [2]. With this approach an individual who is performing exactly as the mean of the group has a score of 100, whereas individual A who is 2 SDs slower than the mean has a score of 70 (calculated as 100 minus $2 \times 15)$ and individual $B$ who is one SD faster than the mean has a score of $115(100$ minus $-1 \times 15)$. In outcomes in which a higher number corresponds to better perfor- mance (e.g., accuracy), the SD is added, not subtracted (e.g., calculated as 100 plus $2 \times 15$ ).

During the fourth step derived from Schouten [3] and Goldsmith [4], it is possible to weigh each dimension. This means, efficacy and safety may be for instance given a higher weight than time and economy of movement. Thus trainees completing their task very fast, but not as intended or not with appropriate safety measures would get a lower score as if all three dimensions were weighed equally. The choice of parameters with more weight and the amount of weight given need careful evaluation and are beyond the scope of this work. For illustrative purposes, we considered in our examples accuracy twice as important as efficiency (time and path length together).

Once the total score has been calculated, it may be further analyzed as any other continuous outcome.

\section{Data examples}

To illustrate the above framework with examples from VR assessment, we re-analyzed the data of two previously published VR studies $[5,6]$.

In the first study, nine children with low and 23 with high experience in videogames, 20 residents and 14 board-certified surgeons were compared for VR laparoscopy performance. A total of 23 outcomes of six basic VR tasks in the three dimensions accuracy (e.g., accuracy of target hits, of cutting and of electrocautery, safe retraction), time and instrument path length (i.e., economy of movement) were considered [5]. Each task was carried out twice.

In the second study, surgical novices were randomized to self-controlled basic VR training ("free training", $n=32$ ) versus VR training based on peer-group-derived benchmarks ("structured training", $n=34$ ). Thereafter the two training groups were compared with each other and with a group of three experts during minimally 60 iterations of simulated cholecystectomy [6]. Five outcomes in the three dimensions accuracy (safe cautery and serious complications), time and instrument path length were measured.

In both studies, the LAP Mentor ${ }^{\mathrm{TM}}$ laparoscopic modules were used (3D Systems, Simbionix Products). Figure 1 illustrates three simulator tasks: a basic VR cutting task, as used in the first data example, and the dissection and clipping steps during simulated cholecystectomy, as carried out in the second data example.

\section{Statistical analyses}

Using the above algorithm and the mean of task repetitions, we calculated the total performance score with accuracy considered twice as important as efficiency. We then analyzed the total performance score further as any other continuous outcome may be analyzed.

We used analysis of variance to compare the different participant groups in each study. For each analysis, we 

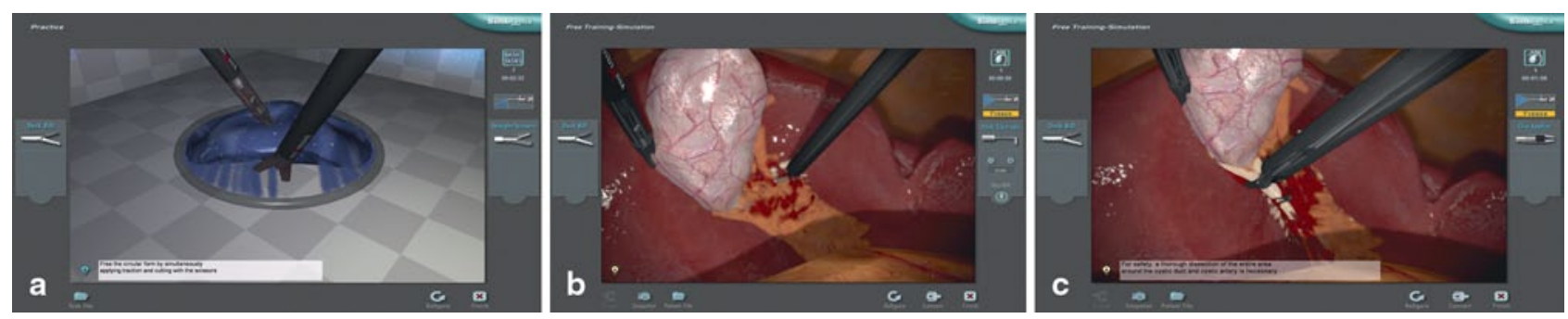

Fig. 1 Virtual reality simulator task (LAP Mentor ${ }^{\mathrm{TM}}$, 3D Systems, Simbionix Products) a Basic tasks module: Cutting (Basic task number 7) b Laparoscopic cholecystectomy full procedure module: Separation c Laparoscopic cholecystectomy full procedure module: Clipping

calculated estimates (with simultaneous $95 \%$ confidence intervals) of the difference in total performance score for all pairwise comparisons. We used Tukey's honest significant difference to adjust the confidence intervals [7].

For analyses and graphics, we used R version 3.0.1 (R Foundation for Statistical Computing, Vienna, Austria) and the $\mathrm{R}$ add-on package lattice version $0.20-24$ [8].

\section{Results}

\section{Example 1: VR laparoscopy performance of} children, residents and board-certified surgeons [5]

Our re-analysis of the data confirmed that video gaming in children improves performance on a VR trainer, but children were outperformed by residents and boardcertified surgeons performed best, as shown in the total performance score (Fig. 2).

\section{Example 2: VR cholecystectomy performance of the free training group, structured training group and experts [6]}

With repeated measures over time summarized into a single mean summary measure per participant, VR cho- lecystectomy performance gradually increased from free training to structured training and experts (Table 1).

\section{Discussion}

We present an algorithm for combining multiple VR outcome metrics into summary measures of the same dimension, and integrating the dimension summary measures into a meaningful total score. We herewith address the risk of selective outcome reporting and multiplicity issues.

When analyzing and reporting multiple outcomes, three different strategies are possible, the choice depending on the study goal and setting: (1) reporting all measured metrics with the challenge of how to address multiplicity (for example opt for graphical display only or control for multiple testing $[9,10])$, (2) reporting part of them with the risk of selective outcome reporting, if not pre-specified in the analysis plan or (3) summarizing multiple outcomes to mean summary measures within dimensions or into a total score, as presented here.

Our approach of is computationally simple and straightforward. The framework may be applied to any VR simulator.

Once the mean summary measures for each dimension or the total score are calculated, they can be ana-
Fig. 2 Boxplots comparing children with low $(n=9)$ and high $(n=22)$ experience in videogames ( $L E$ and $H E$ ), residents $(n=20)$ and boardcertified surgeons $(n=14)$ (Board-cert.) [5] for total virtual reality (VR) performance with accuracy considered twice as important as efficiency

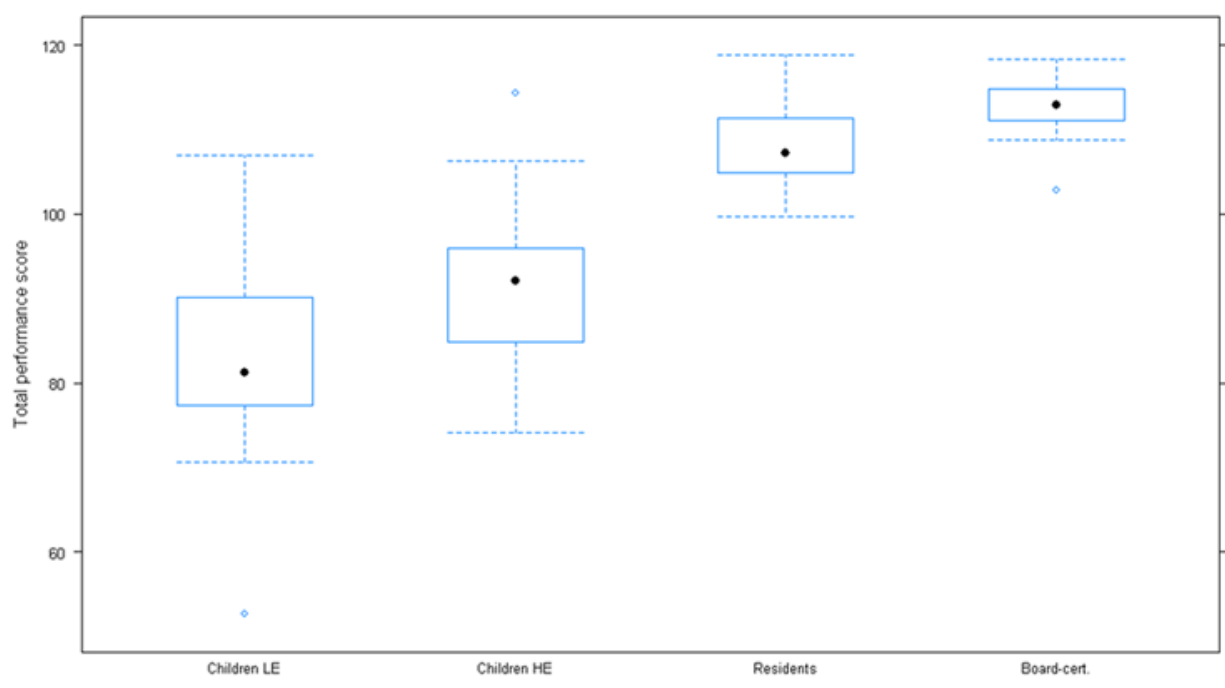


Table 1 Difference in virtual reality (VR) cholecystectomy performance between the three participant groups: free training $(n=31)$, structured training $(n=34)$ and experts $(n=3)$ [6]

\begin{tabular}{|c|c|c|c|c|}
\hline $\begin{array}{l}\text { Difference in VR cholecystectomy performance } \\
\left(95 \% \text { simultaneous }^{\mathrm{a}} \mathrm{Cl}\right)\end{array}$ & Accuracy & Time & Path length & Total \\
\hline Structured training-Free training & $4.2(-3.8,12.3)$ & $-7.1(-15.5,1.3)$ & $-9.1(-17.0,-1.2)^{\star}$ & $5.9(-0.7,12.4)$ \\
\hline Experts-Free training & $11.6(-8.1,31.3)$ & $-25.3(-45.7,-4.8)^{\star}$ & $-22.9(-42.2,-3.7)^{\star}$ & $16.7(0.7,32.7)^{\star}$ \\
\hline Experts-Structured training & $7.3(-12.3,27.0)$ & $-18.2(-38.6,2.1)$ & $-13.8(-33.9,5.4)$ & $10.8(-5.1,26.8)$ \\
\hline $\begin{array}{l}C l \text { confidence interval } \\
{ }^{*} p<0.05 \\
\text { aTukey honest significant differences [7] }\end{array}$ & & & & \\
\hline
\end{tabular}

lyzed further as any other continuous outcome. The units are SDs of the original outcome metrics and the dimension mean summary measures, respectively and the score has been inflated by 15 .

Our investigation presents limitations. First, although the framework is applicable to any simulator, we did not investigate as to whether one individual trainee ends up with a similar total score when running tasks on two different simulators. Second, we did not address the choice of weights of the dimensions, which needs a more indepth discussion and evaluation of the outcomes measured and their translation into clinical outcomes in real life settings.

In conclusion, when analyzing and reporting VR outcome data, the use of standardized, statistically sound, and transparent methods is crucial. We describe an algorithm to combine the evidence from multiple outcome metrics into a total score. As further research, test-retest reliability of the mean summary measures and the total score on the same simulator, followed by test-retest reliability on different simulators should be explored. The ultimate goal of this work and of future research is to enhance the validity of VR-related research reports.

\section{Acknowledgments}

This research was supported by the Swiss National Science Foundation (grant number 32003B-120722). The Basel Institute for Clinical Epidemiology and Biostatistics is supported by grants from Santésuisse and from the Gottfried and Julia Bangerter-Rhyner-Foundation. The funding sources had no involvement in study design, data collection, analysis and interpretation, writing the report and the decision to submit the manuscript for publication.

This work is part of an academic thesis at the University of Basel, Switzerland.

\section{Conflict of interest}

There are no conflicts of interest to declare. Rachel Rosenthal is an employee of F. Hoffmann-La Roche Ltd. since
May 01, 2014. The present study was conducted before Rachel Rosenthal joined F. Hoffmann-La Roche Ltd. and has no connection to her employment by the company. Rachel Rosenthal continues to be affiliated with the University of Basel.

\section{References}

1. Nagendran M, Gurusamy KS, Aggarwal R, Loizidou M, Davidson BR. Virtual reality training for surgical trainees in laparoscopic surgery. Cochrane Database Syst Rev. 2013;8:CD006575.

2. Streiner DL, Norman GR. From items to scales. In: Streiner DL, Norman GR, editors. Health measurement scalesa practical guide to their development and use. 4th ed. Oxford: Oxford University press; 2008. p. 148.

3. Schouten HJ. Combined evidence from multiple outcomes in a clinical trial. J Clin Epidemiol. 2000;53(11):1137-44.

4. Goldsmith $\mathrm{CH}$, Smythe HA, Helewa A. Interpretation and power of a pooled index. J Rheumatol. 1993;20(3):575-8.

5. Rosenthal R, Geuss S, Dell-Kuster S, Schafer J, Hahnloser D, Demartines N. Video gaming in children improves performance on a virtual reality trainer but does not yet make a laparoscopic surgeon. Surg Innov. 2011;18(2):160-70.

6. von Websky MW, Raptis DA, Vitz M, Rosenthal R, Clavien PA, Hahnloser D. Access to a simulator is not enough: the benefits of virtual reality training based on peer-groupderived benchmarks - a randomized controlled trial. World J Surg. 2013;37(11):2534-41.

7. Tukey JW. The problem of multiple comparisons (unpublished manuscript). In: Braun $\mathrm{H}$, editor. The collected works of John W. Tukey VIII. Multiple comparisons. New York: Chapman \& Hall; 1953. pp. 1948-83.

8. Sarkar D. Lattice: multivariate data visualization with $\mathrm{R}$. NY: Springer; 2008.

9. Neuhauser M. How to deal with multiple endpoints in clinical trials. Fundam Clin Pharmacol. 2006;20(6):515-23.

10. Sterne JA, Davey SG. Sifting the evidence-what's wrong with significance tests? BMJ. 2001;322(7280):226-31. 